\title{
Sibylle von Küchler: Ein persönlicher Nachruf auf eine große Hamburger Juristin
}

\section{Renate Damm}

Rechtsanwältin, Ehrenpräsidentin des djb, Hamburg

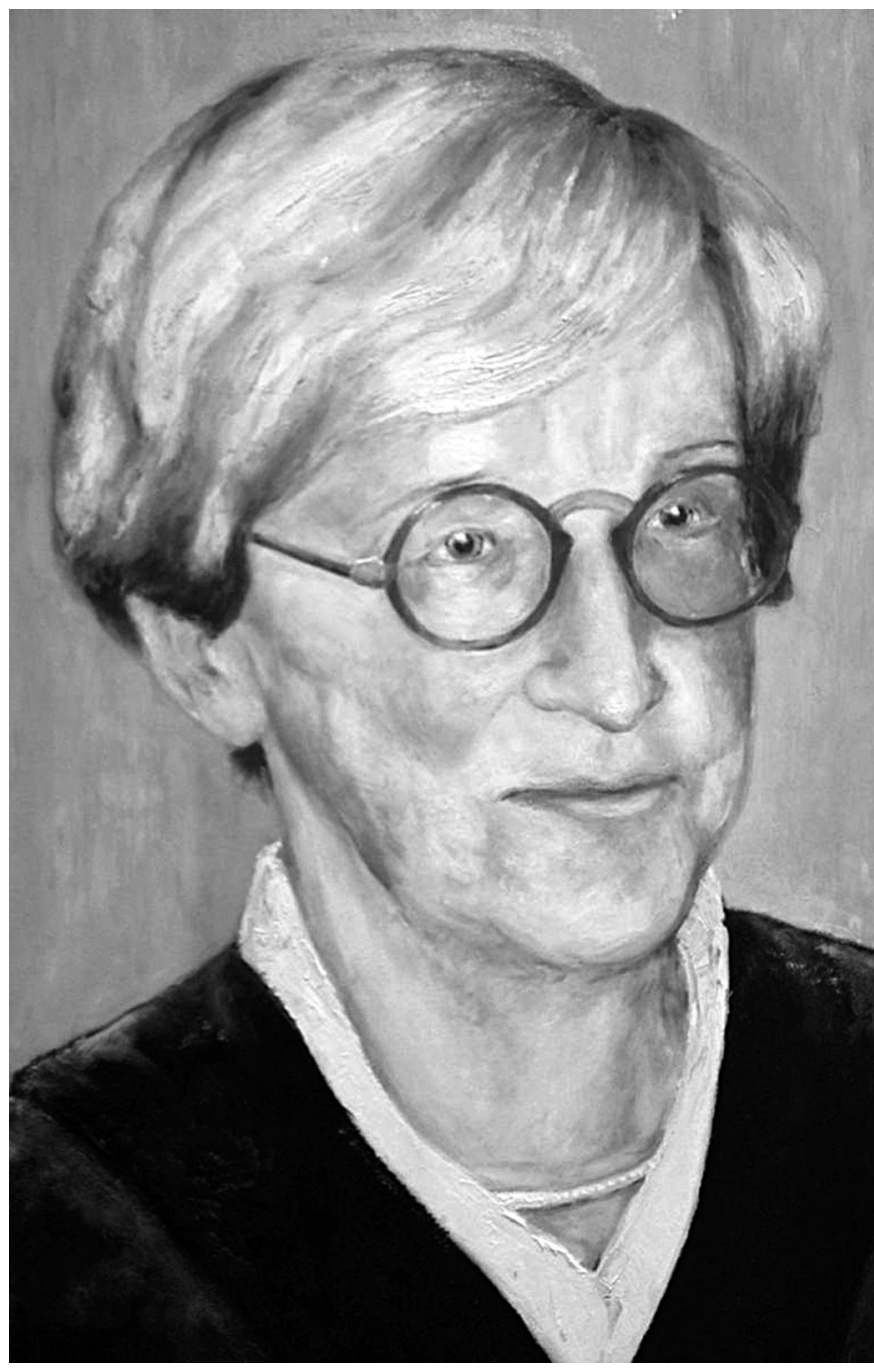

Rechtsanwalt Sibylle von Küchler starb im Alter von 96 Jahren am 25. August 2014 in Hamburg.

Als Referendarin begegnete ich Sibylle zum ersten Mal im Sommer 1961 im Sitzungssaal der Zivilkammer 15 des Landgerichtes Hamburg. Diese Kammer war damals zuständig für Wettbewerbs-, Warenzeichen- und Presserecht. Der Gerichtssaal war voller Männer in Anwaltsroben. Die erste Sache wurde aufgerufen, der Klägervertreter war noch nicht da, plötzlich ging die Tür auf und eine elegante Frau in einem himbeerrotem Cocktailkleid mit einem breitrandigen Strohhut auf dem Kopf und einer Anwaltsrobe über dem Arm trat ein. Sie hängte in aller Ruhe den Strohhut an den Gardarobenhaken, zog die Robe über und trat vor den Richtertisch auf der Klägerseite. „Entschuldigung, aber ich muss anschließend sofort zu einem Empfang ins Rathaus“, erklärte sie ihr wenig anwaltliches Outfit. Ich war schwer beeindruckt. Der Vorsitzende Richter nickte verständnisvoll und die Verhandlung konnte beginnen. Es ging um ein sehr kompliziertes Warenzeichenverfahren. Sibylle von Küchler, die zu dieser Zeit noch Weber hieß, erläuterte den Prozessgegenstand in aller Ruhe und ich hatte den Eindruck, dass auch alle anwesenden männlichen Anwälte aufmerksam zuhörten.

Während meiner Ausbildung in dieser Spezialkammer habe ich Sibylle fast in jeder mündlichen Verhandlung erlebt. Sie war damals wohl die einzige Frau, die als weiblicher Rechtsanwalt, dort auftrat. (Die Berufsbezeichnung „Rechtsanwalt“ war die gesetzlich vorgeschriebene Bezeichnung auch für Frauen.)

Irgendwann traute ich mich, sie anzusprechen. Ein Bekannter von mir hatte rechtliche Probleme mit einem Warenzeichen. Er hatte Schwimmflügel für Kinder aus Plastik entwickelt, weil sein eigener kleiner Sohn fast in einem Pool ertrunken wäre. Diese Schwimmflügel waren warenzeichenrechtlich geschützt, wurden aber als Kopien auf den Markt gebracht. Sibylle übernahm das Mandat und so begann unsere Freundschaft. Diese wurde durch meine eigene spätere Tätigkeit als Justitiarin im Axel Springer Verlag vertieft.

Helene Marianne Sibylle wurde am 7. Mai 1918 in Hamburg als drittes Kind des Rechtsanwaltes Prof. Dr. Gustav Walther Fischer und seiner Ehefrau Katharina Luise Mathilde geboren.

Ihr Vater war neben seiner Tätigkeit als Rechtsanwalt außerplanmäßiger Professor für Urheberrecht und Zivilprozessrecht an der Universität Hamburg. Er war standesrechtlich aktiv als Mitglied im Vorstand der Hanseatischen Rechtsanwaltskammer und des Deutschen Anwaltvereins. Nach 1945 wurde er Präsident der Rechtsanwaltskammern in der Britischen Zone und von der britischen Militärverwaltung als Vertreter der Hamburger Universität in die Hamburger Bürgerschaft berufen. Von 1946 bis 1949 war er Fraktionsvorsitzender der CDU in der Bürgerschaft. Die Mutter war Lehrerin, übte ihren Beruf nach der Eheschließung 1909 aber nicht mehr aus. Sibylle hatte zwei ältere Brüder, die beide im zweiten Weltkrieg fielen.

Sibylle machte ihr Abitur 1936 in der Klosterschule Hamburg, ein Realgymnasium für Mädchen. Danach musste sie kochen und nähen lernen und als Voraussetzung für ein Studium den Arbeitsdienst absolvieren. Zusammen mit ihrem Bruder Joachim begann sie 1937 an der Hamburger Universität Jura zu studieren. Sie wurde dabei ausdrücklich von ihrer Mutter unterstützt.

Nachdem der Bruder zur Wehrmacht eingezogen worden war, unterbrach sie ihr Studium und besuchte von Herbst 1939 bis Frühjahr 1940 die Handelsschule. Danach nahm sie das Studium wieder auf und legte am 8. Dezember 1941 die erste große Staatsprüfung vor dem Justizprüfungsamt bei dem Hanseatischen Oberlandesgericht ab. 
Zuvor, im Januar 1941 heiratete sie den Oberleutnant Günther Pogge. Im Dezember 1942 gebar Sibylle ihre Tochter Christa. Nachdem die Familie im Juli 1943 ausgebombt war, fand sie Unterschlupf bei einer Tante im Harz und kehrte erst nach Kriegsende nach Hamburg zurück.

Endlich, am 10. Dezember 1945, konnte sie ihre Referendarausbildung beginnen. Im Rahmen der Ausbildung gelang Sibylle im April 1946 die Einstellung als Sekretärin im Werbefachverband, der seinen Sitz in der Anwaltskanzlei ihres Vaters hatte. Diese Kanzlei war bereits 1895 von Dr. Ruben Pels gegründet worden. Sie war eine führende Kanzlei u.a. im gewerblichen Rechtsschutz. Prof. Dr. Martin Wassermann, einer der Mitbegründer der Hamburger Universität und Dr. Walther Bußmann wurden u.a. Partner. Sibylle war unheimlich stolz darauf in dieser Kanzlei arbeiten zu können.

Am 2. September 1949 konnte Sibylle endlich vor dem Zentralprüfungsamt für die britische Zone Hamburg die große juristische Staatsprüfung ablegen und danach zunächst als Assessorin und nach einem Jahr als Rechtsanwältin in der Kanzlei ihres Vaters tätig werden. Wie alle Berufsanfänger musste Sibylle sich erst einmal ihren Platz erkämpfen. Sie wollte im Bereich des gewerblichen Rechtsschutzes arbeiten und erreichte dieses Ziel alsbald.

In der Zwischenzeit war Sibylle von ihrem ersten Ehemann geschieden worden und heiratete im August 1949 Dr. iur. Renatus Weber. Am 4. Juli 1950 wurde der gemeinsame Sohn Johann Peter-Michael René geboren.

Dr. Weber trat auch in die Kanzlei ihres Vaters als Sozius ein, war aber in erster Linie politisch tätig. Er war Schriftführer des Verfassungsausschusses und Berichterstatter bei der Schaffung der Hamburger Verfassung und ihr Kommentator, Mitglied der Hamburger Bürgerschaft von 1949 bis 1959, als Senator von 1953 bis 1957 Bevollmächtigter Hamburgs beim Bund und Vorsitzender des Rechtsausschusses des Bundesrats. Danach wurde Dr. Weber Ministerialrat im Bundesministerium für Angelegenheiten des Bundes und der Länder.

Sibylle hatte schon als junges Mädchen nach dem Tod ihrer Mutter (1941) ihren Vater zu vielen offiziellen politischen und gesellschaftlichen Ereignissen begleitet, sodass es für sie keinerlei Problem war, als Ehefrau von Dr. Weber Repräsentationspflichten zu übernehmen. So lernte Sibylle die maßgeblichen Politiker in Bonn von Bundespräsident Heuss bis Bundeskanzler Adenauer kennen. Wichtiger war ihr aber ihre eigene anwaltliche Tätigkeit, weswegen sie nicht nach Bonn umgezogen, sondern ihren Beruf weiter aus Hamburg heraus praktiziert hat. So kam es später zur Trennung.

Im Oktober 1961 heiratete Sibylle den Rechtsanwalt Carl von Küchler, schied aus der Kanzlei Bußmann, Droste, Weber aus und begründete mit ihm eine neue Anwaltskanzlei. Sibylle war weiterhin in ihrem Spezialgebiet des gewerblichen Rechtsschutzes tätig. 1970 wurde auch diese Ehe geschieden, die Kanzlei wurde aber noch bis etwa 1978 gemeinsam weiter ausgeübt. Schließlich kam es zu einer neuen Bürogemeinschaft u.a. mit Dr. Marina Todtenhaupt-Puttfarken (langjährige Schatzmeisterin des djb). Ab 1992 führte Sibylle von Küchler ihre Praxis allein.
Erst im Jahr 2012 hat Sibylle von Küchler ihre Tätigkeit als Rechtsanwältin eingestellt und dies der Kammer mitgeteilt. So endete ihre Zulassung zum 31. Juli 2012. Zu ihrer Zufriedenheit konnte sie die Berufsbezeichnung „Rechtsanwalt“ weiterhin führen, weil ihr dies die Rechtsanwaltskammer auf ihren Antrag gestattet hatte.

Zum 31. Dezember 2012 beendete Sibylle von Küchler ihre Mitgliedschaft im Hamburgischen Anwaltverein und in der Deutschen Landesgruppe der Internationalen Vereinigung für den Schutz des geistigen Eigentums (IPPI). Mitgliedsnummer 384 ! Bis zu ihrem Lebensende blieb sie Mitglied in der Deutschen Vereinigung für gewerblichen Rechtsschutz (Grüner Verein) und im Deutschen Juristinnenbund e. V. (djb).

Viele Tagungen und Veranstaltungen haben wir gemeinsam erlebt, ernsthaft gearbeitet, miteinander gestritten und miteinander gelacht.

Sibylle von Küchler zeichnete eine große Hilfsbereitschaft aus, die ich selbst erleben durfte. 1985, ich war gerade Chefjustitiarin geworden, hatte ich wegen einer Aktion „Bild kämpft für Sie“ erheblichen Ärger mit dem Hamburgischen Anwaltverein. Ein Verfahren wegen Verstoß gegen das Rechtsberatungsmissbrauchgesetz war eingeleitet worden. Sibylle bot mir an, dass sie unter dem Pseudonym „Helen Fischer“ die Leseranfragen anonymisiert beantworten würde. So geschah es und der Hamburger Anwaltverein gab sich damit zufrieden. Später entdeckte der Verein, dass es sich auch um eine gute PR-Maßnahme handeln könnte und arbeitete mit der BILDRedaktion zusammen.

Sibylle war sehr glücklich, dass ihre Tochter Christa auch Juristin wurde. Diese war unter ihrem Ehenamen Seeliger Familienrichterin. Natürlich ist sie Mitglied im djb (in der Bonner Untergruppe). Besonders stolz war Sibylle darauf, dass auch beide Töchter von Christa Seeliger Juristinnen sind. Dorian ist in der öffentlichen Verwaltung tätig und Daniela ist Partnerin in einer großen Kanzlei. Ihr Gebiet ist das Kartellrecht.

Sibylle war auch sehr glücklich, insbesondere in den letzten Jahren, ihren Sohn und die Zwillingsenkel Georg und Justus sowie die Familien ihrer Enkelinnen Svenja und Claire um sich zu haben, letztere lebte längere Zeit im gleichen Haus. Ein besonderer Lichtblick waren die Urenkel, deren Aufwachsen sie mit Freude verfolgt hat, ob aus Hamburg oder zu Besuch aus Köln und Trier. So war sie bis zuletzt Oberhaupt und aktiv von allen in das Familiengeschehen eingebunden, kümmerte sich, war interessiert, gab auf ihre Art Liebe und Zuneigung. Sie überstand auch schwere Krankheiten und ließ sich ihre Freude am Leben nicht nehmen.

Irgendwann einmal hatte sie mich in meinem Haus in Hamburg-Bergstedt besucht und ich musste ihr Milch zum Kaffee aus einem nicht zu dem übrigen Geschirr passenden Kännchen anbieten. Mein „Early Morning“ Geschirr war nicht mehr nachzukaufen. Jahre später erhielt ich ein kleines Päckchen von Sibylle. Sie hatte das passende Milchkännchen auf einem Trödelmarkt entdeckt.

Dieses kleine Kännchen wird mich immer an Sibylle erinnern. 


\section{Geburtstage}

(Oktober bis Dezember 2014)

\section{Jahre}

- Prof. Angelika Meeth-Milbradt Professorin (FH)

Dresden

\section{Jahre}

- Helga Fleckstein

Rechtsanwältin/Dozentin Berlin

- Dr. Katrin Lausen

Rechtsanwältin

Freiburg

\section{Jahre}

- Hilke Amthor

Vorsitzende Richterin am

Oberlandesgericht i.R.

Kronberg

- Reglindis Böhm

Landgerichtspräsidentin a.D.

Rockenberg

\section{- Christa Schulte}

Richterin am Amtsgericht i.R.

Karlsruhe

\section{Verstorben}

(Juli bis September 2014)

\section{- Anneliese Glorius}

Rechtsanwältin i.R.

Aachen

im 91. Lebensjahr

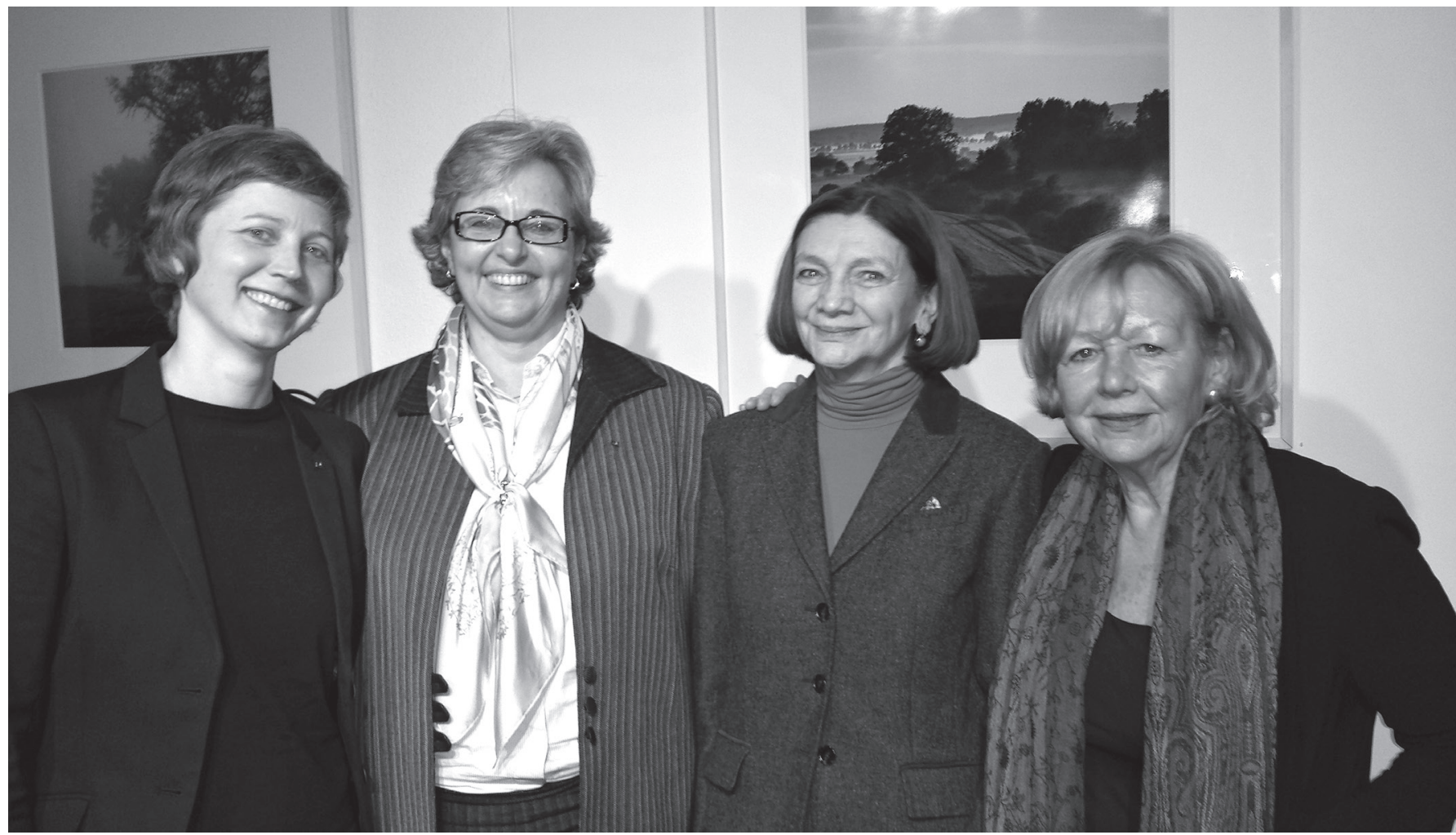

A Vier (ehemalige) Vorsitzende des Landesverbands Berlin im djb: Rechtsanwältin Sabine Wildfeuer, Rechtsanwältin Prof. asoc. Dr. Jutta Glock, Rechtsanwältin und Notarin Jutta Wagner, Rechtsanwältin Ursula Raue (v.l.n.r.). Ursula Raue war bereits bei der Gründung der „Untergruppe Berlin“ am 28. September 1983 dabei und wurde dessen erste Vorsitzende (zunächst 1983/1984, das Amt übte sie erneut von 1988 bis 1992 aus). 1989 wurde Ursula Raue außerdem in den djb-Bundesvorstand gewählt, 1993 bis 1997 war sie dann dessen 1. Vorsitzende. Jutta Wagner war ebenfalls mehrere Jahre (1998 bis 2004) Vorsitzende des Landesverbands Berlin und um anschließend (2005 bis 2011) das Amt der Präsidentin des djb zu übernehmen. 